\title{
Stochastic Sensitivity Analysis and Control for Ecological Model with the Allee Effect
}

\author{
L. Ryashko *, I. Bashkirtseva
}

Institute of Mathematics and Computer Sciences, Ural Federal University, 620083 Ekaterinburg, Russia

\begin{abstract}
In this paper, we discuss a problem of the analysis and prevention of catastrophic shifts in ecosystems with stochastic environment. For the solution of this problem in models of ecological dynamics, a new approach based on the stochastic sensitivity functions technique is suggested and applied to a stochastically forced predator-prey model with the Allee effect. For this population model, we analyze a phenomenon of noise-induced extinction using the method of confidence domains. By controlling those domains we provide a stable coexistence of both species and prevent the noise-induced extinction.
\end{abstract}

Keywords and phrases: stochastic sensitivity, ecological models, population dynamics, stabilization

Mathematics Subject Classification: 60H10, 92D40, 93E15

\section{Introduction}

Environmental noise is an inevitable attribute of dynamics of any ecosystem [17]. Abrupt catastrophic shifts in ecosystems can be caused by even small deterministic and stochastic disturbances. From theoretical point of view, such shifts can be explained by the coexistence of alternative stable states, nonuniformity of phase portraits, and sensitivity of boundaries of basins of attraction for corresponding dynamic models $[18,21]$.

Under the random disturbances, the system can leave a deterministic basin of attraction, cross the separatrix and continue to operate near another attractor. An interplay between nonlinearity, multistability and stochasticity is a challenging problem of the modern theoretical ecology. For mathematical modeling of stochastic dynamics of ecological systems, the stochastic differential equations are widely used $[1,6,9,22-24]$.

The most detailed probabilistic description of the stochastic flows in these models is given by Kolmogorov-Fokker-Planck (KFP) equation. However, it is hard to use this equation directly, even in $2 \mathrm{D}$ case. Usually, statistical properties are studied by the direct numerical simulation. However, in a parametrical analysis, this method is highly time-consuming. Under these circumstances, one can use

\footnotetext{
${ }^{*}$ Corresponding author. E-mail: lev.ryashko@urfu.ru
} 
analytical approximations and asymptotics. For the approximation of KFP solutions, the quasipotential method is well known $[10,12]$. In the framework of this approach, a stochastic sensitivity function (SSF) technique and geometrical description of stochastic attractors via confidence domains were proposed in $[2,15]$. SSF technique was successfully applied for the stabilization of stochastic attractors and suppression of chaos $[3,19]$, and also for the analysis of noise-induced excitement in a prey-predator plankton system [20].

Along with the problem of the analysis of unwanted shifts in live systems, control problems for ecosystems are also highly relevant [11]. Ecosystem management attracts attention of many researchers [8]. Stable regimes of dynamics of ecosystem are provided by intrinsic natural feedback mechanisms of regulation $[4,5,7]$.

If such mechanisms wear out or are destroyed under various disturbances then this desired stabilization can be achieved by additional artificial feedbacks. In present paper we show how SSF technique and confidence domains method can be applied for the analysis and prevention of the undesirable shifts in ecosystems. To capture main ideas of our approach in a transparent way, we choose the simple predatorprey population model with the Allee effect [16].

In Section 2, main features of the uncontrolled model are discussed such as bistability, noise-induced transitions across separatrix and extinction.

In Section 3, a mathematical background for the analysis and control of stochastic equilibria via SSF technique is briefly presented.

In Section 4 and 5, we discuss how this theory can be applied to the parametrical analysis and control of the stochastic predator-prey population model.

\section{Stochastic Population Model with the Allee Effect}

Consider a general predator-prey system described by two differential equations

$$
\left\{\begin{array}{l}
\frac{d p}{d \tau}=g(p)-f(p) z \\
\frac{d z}{d \tau}=a f(p) z-b z
\end{array}\right.
$$

where $p$ and $z$ are densities of the prey and predator at time $\tau$. The function $g(p)$ describes an intrinsic prey growth, $f(p)$ is a predator trophic response, $a$ is a food utilization coefficient, $b$ is a predator mortality. A recent review on the predator-prey models with the Allee effect was presented in [13].

In present paper, we consider the predator trophic response of Holling type II, and for the function $f(p)$ the following parameterization is used [14]

$$
f(p)=\frac{A p}{B+p},
$$

where $B$ is the half-saturation prey density, and $A$ is the maximum predation rate.

A strong Allee effect for prey population dynamics can be modeled by the function $g(p)$ in the following standard parametrization:

$$
g(p)=c p\left(p-p_{0}\right)(K-p),
$$

where $c$ is the prey growth rate, $K$ is the prey carrying capacity, $p_{0}$ is the prey survival threshold. The value $p_{0}: 0<p_{0}<K$, is a control parameter for the Allee effect intensity.

In dimensionless variables $x=p / K, y=z /(a K), t=(A a K / B) \tau$, the equation (2.1) can be rewritten as

$$
\left\{\begin{array}{l}
\frac{d x}{d t}=\gamma x(x-\beta)(1-x)-\frac{x y}{1+\alpha x} \\
\frac{d y}{d t}=\frac{x y}{1+\alpha x}-\delta y .
\end{array}\right.
$$



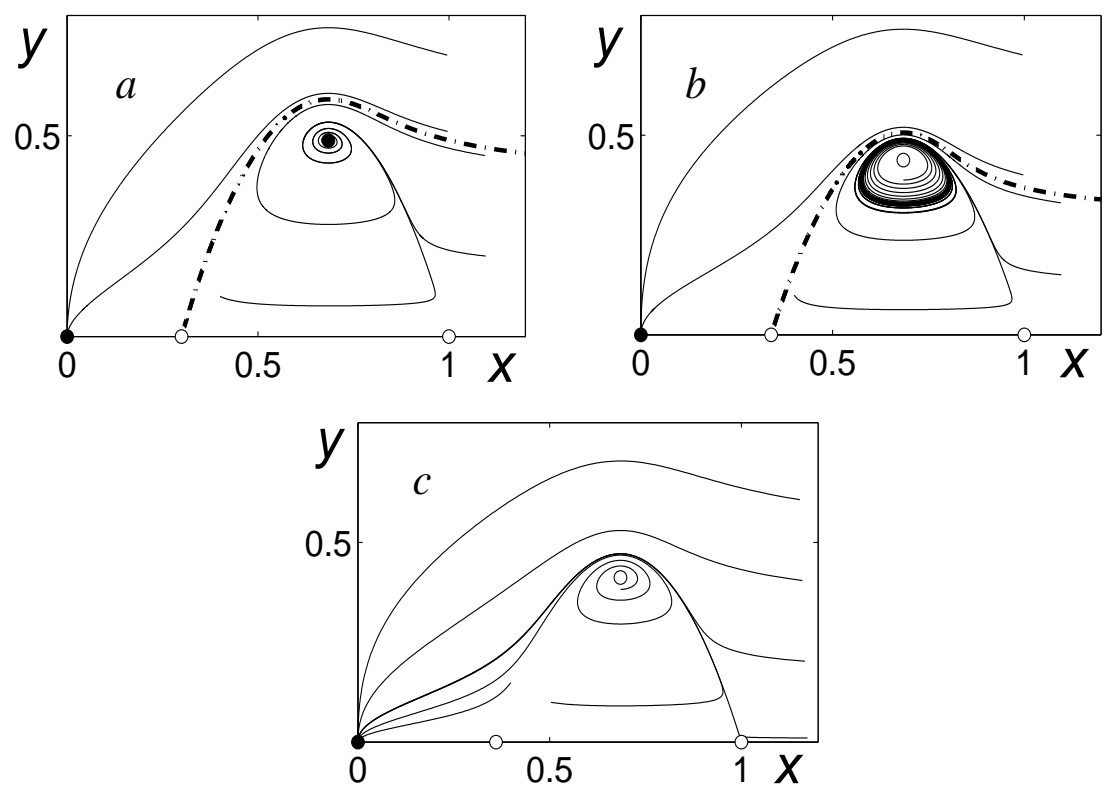

FiguRE 1. Phase portraits of the deterministic system with separatrices (dash-dotted) for a) $\beta=0.3$; b) $\beta=0.34$; c) $\beta=0.36$.

Here, $\alpha=K / B, \beta=p_{0} / K, \gamma=c K B /(a A), \delta=b B /(a A K)$ are dimensionless parameters. This simple two-dimensional model is quite representative for the demonstration of noise-induced ecological shifts (extinction) and discussion how to prevent these shifts. Actually, this model exhibits bistability, sensitivity of basins of attraction to variation of parameters, local and global bifurcations.

The deterministic system $(2.2)$ has four equilibria $M_{1}(0,0), M_{2}(\beta, 0), M_{3}(1,0), M_{4}(\bar{x}, \bar{y})$, where

$$
\bar{x}=\frac{\delta}{1-\alpha \delta}>0, \bar{y}=\gamma(1+\alpha \bar{x})(\bar{x}-\beta)(1-\bar{x})>0
$$

for $\beta<\frac{\delta}{1-\alpha \delta}<1$. The trivial equilibrium $M_{1}$ is stable and equilibria $M_{2}, M_{3}$ are unstable for any parameters.

In this paper, we fix the following set [16] of parameters: $\alpha=0.5, \gamma=3, \delta=0.51$ and vary the Allee parameter $\beta \in[0.3,0.38]$. In this $\beta$-interval, the local and global bifurcations occur, and the system $(2.2)$ exhibits three different regimes of dynamics (see Fig. 1).

The non-degenerate equilibrium $M_{4}(\bar{x}, \bar{y})$ corresponds to the coexistence of prey and predator. This equilibrium is stable for $0.3<\beta<\beta_{*}=0.3271$. As the parameter $\beta$ gets over the value $\beta_{*}$ from left to right, the equilibrium $M_{4}(\bar{x}, \bar{y})$ loses stability and the system (2.2) demonstrates auto-oscillations with the limit cycle $\gamma$.

The value $\beta_{*}$ is the Andronov-Hopf bifurcation point. In Fig. 1a, for $\beta=0.3<\beta_{*}$, a separatrix (dashdotted) detaches basins of the attraction of the stable equilibrium $M_{1}(0,0)$ and the stable equilibrium $M_{4}$. For $\beta=0.34>\beta_{*}$ (see Fig. 1b), separatrix detaches basins of the attraction of the stable equilibrium $M_{1}(0,0)$ and the stable limit cycle $\gamma$. Here, $\beta_{*}$ is the point of a local bifurcation. As $\beta$ increases, limit cycle enlarges and at $\beta^{*}=0.35529$ cycle is destroyed: a lower part of the cycle coalesces with the line $y=0$, upper part adheres to the separatrix, and heteroclinic orbit is born. Here, $\beta^{*}=0.35529$ is the point of a global bifurcation. In Fig. 1c, the phase portrait with the single stable equilibrium $M_{1}$ for $\beta=0.36>\beta^{*}$ is shown. 
It follows from this analysis that for $0.3 \leq \beta<\beta^{*}$ the extinction zone is restricted by separatrix from below, and for $\beta^{*}<\beta \leq 0.38$ the extinction zone coincides with the whole first quadrant $x>0, y>0$. So, for $0.3 \leq \beta<\beta^{*}$, the separatrix serves as the boundary between a zone of the positive coexistence of prey with predator and a zone of the total extinction.

a)

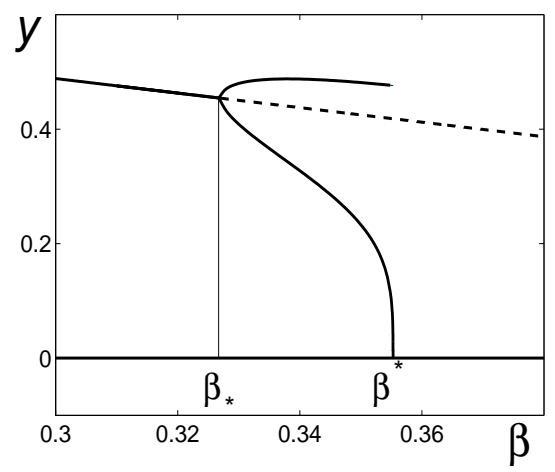

b)

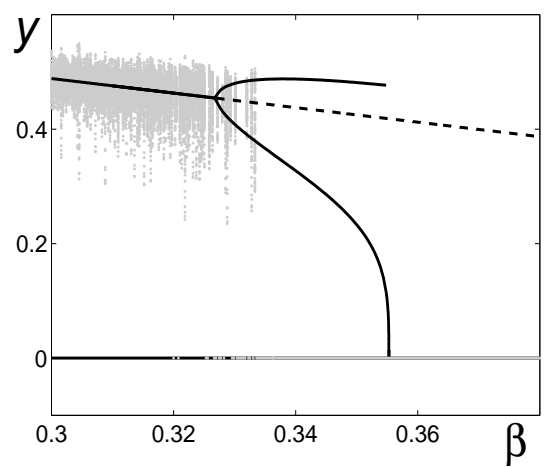

c)

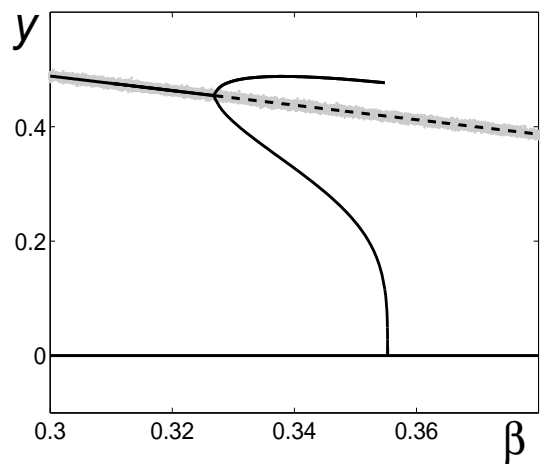

Figure 2. a) Bifurcation diagram of the deterministic system; b) random states (grey) of the system without control and $\varepsilon=0.01, \mathrm{c}$ ) random states (grey) of the system with control and $\varepsilon=0.01, w=0.1$.

In Fig. $2 \mathrm{a}$, for $0.3 \leq \beta \leq 0.38$, attractors of the deterministic system (2.2) are presented. Here, values of $y$-coordinate for the stable equilibria $M_{1}, M_{4}$ and extreme values of variable $y$ for the stable limit cycle are plotted by solid lines. Dashed line corresponds to the $y$-coordinate of the unstable equilibrium $M_{4}$. Here a splitting of upper graph marks the bifurcation point $\beta_{*}$. For $\beta=\beta^{*}$ the limit cycle disappears and for $\beta>\beta^{*}$ the trivial equilibrium $M_{1}$ remains as a single attractor, so the total extinction is inevitable for any initial data.

Along with initial deterministic model (2.2) consider a stochastically forced model

$$
\left\{\begin{array}{l}
\dot{x}=\gamma x(x-\beta)(1-x)-\frac{x y}{1+\alpha x}-\sigma_{1} \gamma x(1-x) \xi_{1}, \\
\dot{y}=\frac{x y}{1+\alpha x}-\delta y-\sigma_{2} y \xi_{2} .
\end{array}\right.
$$

Here $\xi_{1}(t)$ and $\xi_{2}(t)$ are $\delta$-correlated white Gaussian noises satisfying $\left\langle\xi_{i}(t)\right\rangle=0,\left\langle\xi_{i}(t) \xi_{j}(\tau)\right\rangle=\delta(t-$ $\tau) \delta_{i j}, \quad \sigma_{1}, \sigma_{2}$ are intensities of multiplicative noises modelling random disturbances of parameters $\beta$ 
and $\delta$ correspondingly. The stochastic model (2.3) is obtained from the deterministic model (2.2) after replacing: $\beta \rightarrow \beta+\sigma_{1} \xi_{1}, \delta \rightarrow \delta+\sigma_{2} \xi_{2}$.

Here we consider $\sigma_{1}=\sigma_{2}=\varepsilon$.

a)

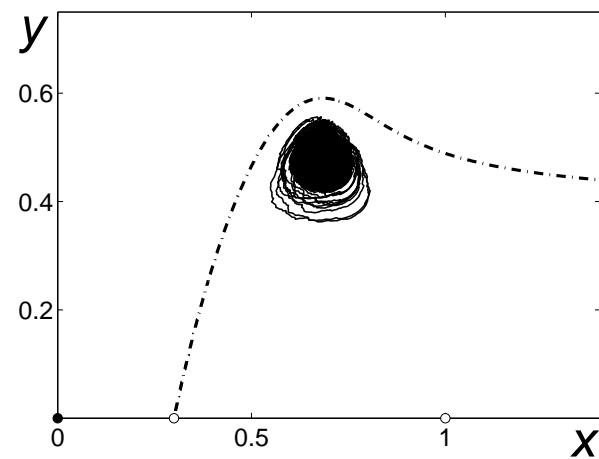

c)

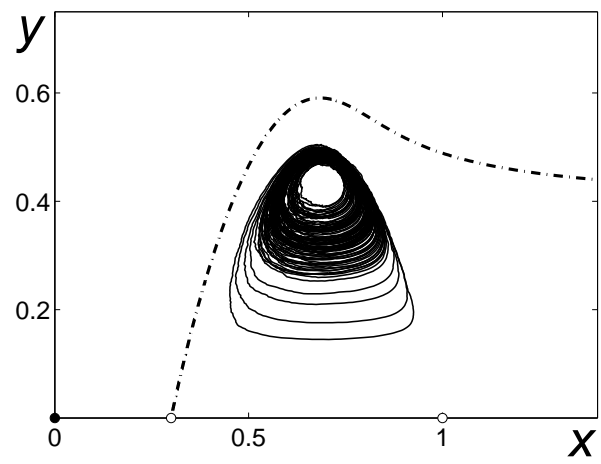

b)

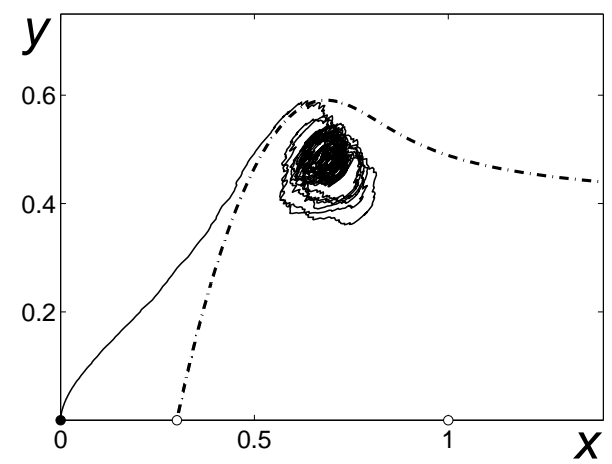

d)

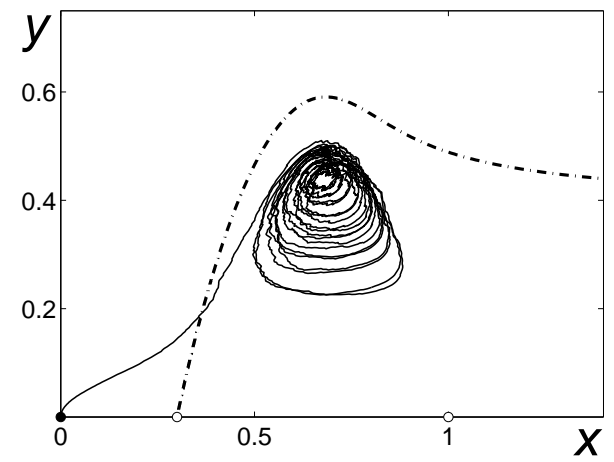

FiguRE 3. Stochastic trajectories for $\beta=0.3$ (top panel) and $\beta=0.34$ (bottom panel): a) non-exit for $\varepsilon=0.01$; b) noise-induced exit for $\varepsilon=0.02$, c) non-exit for $\varepsilon=0.005$; d) noise-induced exit for $\varepsilon=0.01$.

Consider an influence of the parametric noises in the system (2.3) for zone $0.3<\beta<\beta^{*}=0.35529$, where the initial deterministic model predicts the stable stationary or periodic regimes of the coexistence of both species due to the stability of the non-trivial equilibrium $M_{4}$ or limit cycle correspondingly. Under random disturbances, stochastic trajectories of the forced system (2.3) leave deterministic attractors.

For weak noise, due to stability, the random trajectories are concentrated near these attractors (see Fig. 3a for stable equilibrium and Fig. 3c for stable cycle).

As noise intensity increases, a dispersion of the random states is increases too, and the system (2.3) can exhibit qualitative changes of stochastic dynamics. Under the random disturbances, the stochastic trajectory can exit from the neighborhood of the stable attractor, cross the separatrix and pass to $M_{1}$ (see Figs. 3b,d). In Fig. 2b, random states of the system (2.3) with $\varepsilon=0.01$ are presented by grey color. Here, for $t=0$, trajectories started from the equilibrium $M_{4}$ and after transient time period [0,200] were plotted for $t \in[200,300]$. The corresponding time series for coordinate $y(t)$ are presented in Fig. 6 by grey color.

These transitions can be interpreted as a noise-induced extinction of both species in this population. 
In what follows we will show how to use a stochastic sensitivity functions technique to control the dispersion of random states around the equilibrium and prevent the noise-induced extinction. In the next Section, a mathematical background of the stochastic sensitivity functions technique is discussed briefly.

\section{SSF technique for analysis and control of stochastic equilibria}

Consider a nonlinear system of stochastic differential equations

$$
\dot{x}=f(x)+\varepsilon \sigma(x) \xi,
$$

where $\xi$ is an $n$-dimensional Gaussian white noise satisfying $\langle\xi(t)\rangle=0,\langle\xi(t) \xi(\tau)\rangle=\delta(t-\tau) I, I$ is an identity matrix, $\sigma(x)$ is $n \times n$-matrix function of disturbances with intensity $\varepsilon$.

Let $\bar{x}$ be a stable equilibrium of the corresponding deterministic system. Under the random disturbances, due to stabililty of $\bar{x}$, stochastic states of forced system (3.1) are concentrated around $\bar{x}$ and form a stationary probabilistic distribution $\rho(x, \varepsilon)$ (stochastic equilibrium).

For low noise, we use the following Gaussian approximation of $\rho(x, \varepsilon)$ :

$$
\rho(x, \varepsilon) \approx K \cdot \exp \left(-\frac{\left(x-\bar{x}, W^{-1}(x-\bar{x})\right)}{2 \varepsilon^{2}}\right),
$$

based on the theory of the quasipotential [12].

A dispersion (mean-square deviation) of random states around the exponentially stable equilibrium $\bar{x}$ can be approximated by the following formula [19]

$$
\mathrm{E}(x-\bar{x})(x-\bar{x})^{\top} \approx \varepsilon^{2} W .
$$

Here, the matrix $W$ is a unique solution [15] of the matrix equation

$$
F W+W F^{\top}+S=0, \quad F=\frac{\partial f}{\partial x}(\bar{x}), \quad S=\sigma(\bar{x}) \sigma^{\top}(\bar{x}) .
$$

The matrix $W$ is a stochastic sensitivity function (SSF) of the equilibrium $\bar{x}$. The eigenvalues of this matrix are convenient scalar characteristics of the stochastic sensitivity of the equilibrium.

For 2D-case, this matrix characterizes a spatial arrangement and size of the confidence ellipse

$$
\left(x-\bar{x}, W^{-1}(x-\bar{x})\right)=2 \varepsilon^{2} q .
$$

Here $\varepsilon$ is a noise intensity, $q=-\ln (1-P), P$ is a fiducial probability. Let $\lambda_{1}, \lambda_{2}$ are eigenvalues and $v_{1}, v_{2}$ are normalized eigenvectors of the stochastic sensitivity matrix $W$. For the coordinates $\xi_{1}=$ $\left(x-\bar{x}, v_{1}\right), \xi_{2}=\left(x-\bar{x}, v_{2}\right)$, the equation of the confidence ellipse in a basis of $v_{1}, v_{2}$ with the origin at the point $\bar{x}$ can be written in a standard form:

$$
\frac{\xi_{1}^{2}}{\lambda_{1}}+\frac{\xi_{2}^{2}}{\lambda_{2}}=2 \varepsilon^{2} q
$$

Note that if $\lambda_{1} \geq \lambda_{2}$ then a major semi-axis of the confidence ellipse is $\varepsilon \sqrt{2 \lambda_{1} q}$, and minor semi-axis is $\varepsilon \sqrt{2 \lambda_{2} q}$.

Confidence ellipse is a simple geometrical model for the description of a configurational arrangement of random states near stable equilibrium.

Consider a nonlinear controlled stochastic system

$$
\dot{x}=f(x, u)+\varepsilon \sigma(x) \xi,
$$


where $f(x, u)$ is a continuously differentiable vector-function, $u$ is a control input.

It is supposed that the corresponding deterministic system (3.3) (with $\varepsilon=0$ and $u=0$ ) has an equilibrium $\bar{x}$. A stability of $\bar{x}$ is not assumed.

Here, we consider a regulator in the form of linear feedback

$$
u(x)=K(x-\bar{x}) .
$$

To provide an exponential stability of the equilibrium $\bar{x}$ of the closed-loop system (3.3), (3.4), the feedback matrix $K$ has to belong to the following set:

$$
\mathbf{K}=\left\{K \mid \operatorname{Re} \lambda_{i}(F+B K)<0\right\},
$$

where $F=\frac{\partial f}{\partial x}(\bar{x}, 0), B=\frac{\partial f}{\partial u}(\bar{x}, 0)$.

Under the small external disturbances $(\varepsilon \neq 0)$, the random trajectories of the stochastic system (3.3) leave the equilibrium $\bar{x}$. Due to exponential stability, the feedback (3.4) with a matrix $K \in \mathbf{K}$ localizes random states of the system $(3.3),(3.4)$ in the neighborhood of the equilibrium $\bar{x}$ and form a stationary distribution around it.

The aim of the control is to provide an assigned stochastic sensitivity matrix $W$ of this distribution by the corresponding choice of the matrix $K$.

This matrix $W$ is a solution of the equation

$$
(F+B K) W+W(F+B K)^{\top}+S=0 .
$$

In a case of non-singular noises $(\operatorname{det} S \neq 0)$ the solution $W$ is positive defined.

The connection between the assigned matrix $W$ and the feedback matrix $K$ follows from the equation (3.5) which can be rewritten in the form:

$$
\begin{aligned}
& B K W+W K^{\top} B^{\top}+H(W)=0, \\
& H(W)=S+F W+W F^{\top} .
\end{aligned}
$$

A detailed mathematical solution of the problem of synthesis of the stochastic sensitivity matrix can be found in [19]. Here we present basic results:

a) if $\operatorname{rank}(B)=n$ then the matrix $B^{-1}$ exists and for any assigned positive defined matrix $W$ the feedback matrix $K$ can be found as

$$
K=-B^{-1}\left(F+\frac{1}{2} S W^{-1}\right)
$$

b) if $\operatorname{rank}(B)<n$ then attainable matrices $W$ have to satisfy the equation

$$
P H(W) P=0, \quad P=I-B B^{+} .
$$

In this case, one can find a matrix $K$ as the following:

$$
K=-\frac{1}{2} B^{+} H(W)(P+I) W^{-1} .
$$

Here, a sign "+" means a pseudoinversion.

\section{Stochastic Sensitivity of Population Model}

Consider the application of the theory of stochastic sensitivity from Section 3 to the analysis of the stochastic population model (2.3) with $\sigma_{1}=\sigma_{2}=\varepsilon$. For this system,

$$
F=\left[\begin{array}{cc}
f_{11} & f_{12} \\
f_{21} & 0
\end{array}\right], \quad S=\left[\begin{array}{cc}
s_{1} & 0 \\
0 & s_{2}
\end{array}\right],
$$


where

$$
\begin{gathered}
f_{11}=\gamma[(\bar{x}-\beta)(1-\bar{x})+\bar{x}(1-\bar{x})-\bar{x}(\bar{x}-\beta)]-\frac{\bar{y}}{(1+\alpha \bar{x})^{2}}, \\
f_{12}=-\frac{\bar{x}}{1+\alpha \bar{x}}, \quad f_{21}=\frac{\bar{y}}{(1+\alpha \bar{x})^{2}}, \quad s_{1}=\gamma^{2} \bar{x}^{2}(1-\bar{x})^{2}, \quad s_{2}=\bar{y}^{2} .
\end{gathered}
$$

Due to (3.2), for the elements of the stochastic sensitivity matrix $W=\left[\begin{array}{ll}w_{11} & w_{12} \\ w_{12} & w_{22}\end{array}\right]$, the following system holds:

$$
\begin{aligned}
& 2 f_{11} w_{11}+2 f_{12} w_{12}+s_{1}=0 \\
& f_{21} w_{11}+f_{11} w_{12}+f_{12} w_{22}=0 \\
& 2 f_{21} w_{11}+s_{2}=0
\end{aligned}
$$

This system has a solution

$$
w_{11}=\frac{f_{12} s_{2}-f_{21} s_{1}}{2 f_{11} f_{21}}, \quad w_{12}=-\frac{s_{2}}{2 f_{21}}, \quad w_{22}=\frac{1}{2 f_{12}}\left[\frac{f_{21} s_{1}-f_{12} s_{2}}{f_{11}}+\frac{s_{2}}{f_{21}}\right] .
$$

For $\beta=0.3$, we have the following stochastic sensitivity matrix $W=\left[\begin{array}{cc}8.82 & -0.44 \\ -0.44 & 4.73\end{array}\right]$.

Using eigenvalues and eigenvectors of this matrix, we can plot confidence ellipses around the stable equilibrium $M_{4}$ for various values of the noise intensity(see Fig. 4). For sufficiently small noise intensity, the confidence ellipses localized near the equilibrium entirely belong to the basin of the attraction of $M_{4}$. Corresponding random trajectories are concentrated near $M_{4}$.

As the noise intensity increases, the confidence ellipses begin to expand and after crossing the separatrix, they enter the basin of attraction of $M_{1}$. This occupation means that random trajectories of stochastic system with a high probability can leave the basin of attraction of $M_{4}$ and go to the trivial equilibrium $M_{1}$. Noise intensity that corresponds to the intersection of confidence ellipse with separatrix can be used as an estimation of the threshold value $\varepsilon^{*}$. Here $\varepsilon^{*} \approx 0.015$. It is important to take into account this value for the prediction of the potential ecological shifts, namely extinction in this population system.

To protect the population system from unwanted noise-induced ecological shifts leading to the extinction, we will construct a feedback in such a way as to localize a confidence ellipse inside the basin of attraction of the equilibrium $M_{4}$. As shown above, the confidence ellipse for the uncontrolled system with $\varepsilon=0.02$ (see Fig. 4) is too wide. Indeed, this confidence ellipse contains the separatrix and partly occupies the basin of attraction of the trivial equilibrium. Here, the control problem can be reduced to decreasing the size of the confidence ellipse.

\section{Control of Stochastic Sensitivity for Population Model}

Consider now the population model with control

$$
\left\{\begin{array}{l}
\dot{x}=\gamma x(x-\beta)(1-x)-\frac{x y}{1+\alpha x}+u_{1}-\sigma_{1} \gamma x(1-x) \xi_{1}, \\
\dot{y}=\frac{x y}{1+\alpha x}-\delta y+u_{2}-\sigma_{2} y \xi_{2}
\end{array}\right.
$$

where $u_{1}$ and $u_{2}$ are control inputs.

Consider two cases. In the first case (Control I), we have an opportunity to act on both equations of the system (5.1) by inputs $u_{1}$ and $u_{2}$. In the second case (Control II), our action is restricted: we can force the first equation only $\left(u_{2}=0\right)$. 


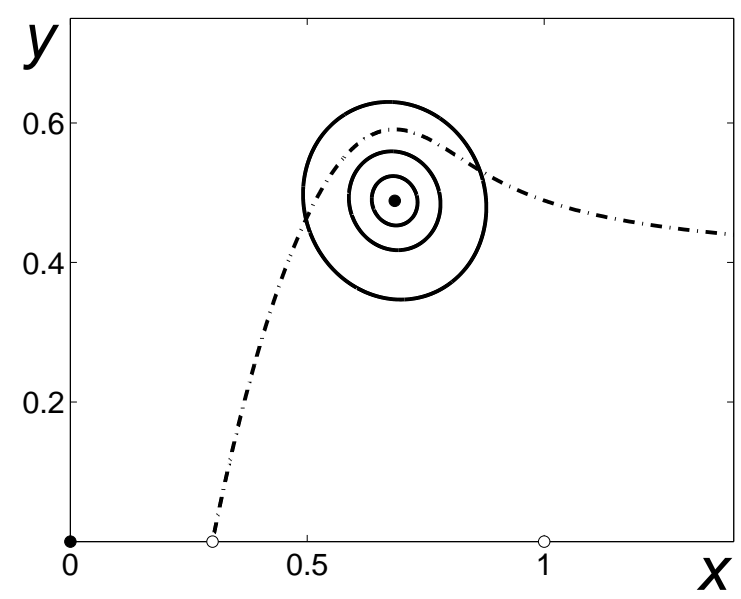

Figure 4. Confidence ellipses for $\beta=0.3$ and $P=0.9$ for the system without control and $\varepsilon=0.005$ (small), $\varepsilon=0.01$ (middle), $\varepsilon=0.02$ (large).

Control I. In this case $B=\left[\begin{array}{ll}1 & 0 \\ 0 & 1\end{array}\right]$. Due to (3.7), a feedback matrix $K$ can be written as

$$
K=-F-\frac{1}{2} S W^{-1}
$$

Note that for the system (5.1), any stochastic sensitivity matrix $W$ is attainable.

To decrease the stochastic sensitivity, we assign the diagonal matrix $W: W=\left[\begin{array}{cc}w & 0 \\ 0 & w\end{array}\right]$ with a sufficiently small value $w$.

Here,

$$
W^{-1}=\frac{1}{w}\left[\begin{array}{ll}
1 & 0 \\
0 & 1
\end{array}\right], \quad K=-F-\frac{1}{2 w}\left[\begin{array}{cc}
s_{1} & 0 \\
0 & s_{2}
\end{array}\right]
$$

So,

$$
k_{11}=-f_{11}-\frac{s_{1}}{2 w}, \quad k_{12}=-f_{12}, \quad k_{21}=-f_{21}, \quad k_{22}=-\frac{s_{2}}{2 w} .
$$

In Fig. 5a, confidence ellipses for $\beta=0.3, P=0.9$, and $\varepsilon=0.02$ are plotted: large ellipse for the system without control, and middle and small ellipses for the system with Control I providing $w=1$ and $w=0.01$, correspondingly.

As one can see a synthesis of the small stochastic sensitivity results in the small size of confidence ellipse and localization of random states near the equilibrium $M_{4}$.

Control II. In this case

$$
B=\left[\begin{array}{l}
1 \\
0
\end{array}\right], \quad B^{+}=\left[\begin{array}{ll}
1 & 0
\end{array}\right], \quad P=\left[\begin{array}{ll}
0 & 0 \\
0 & 1
\end{array}\right], \quad P H P=\left[\begin{array}{cc}
0 & 0 \\
0 & 2 f_{12} w_{12}+s_{2}
\end{array}\right] .
$$

If follows from (3.8) that $w_{12}=-\frac{s_{2}}{2 f_{21}}$. This means that using this type of control, we can not assign this element of the required stochastic sensitivity matrix $W$. Note that here $w_{12}$ is the same as for the 
a)

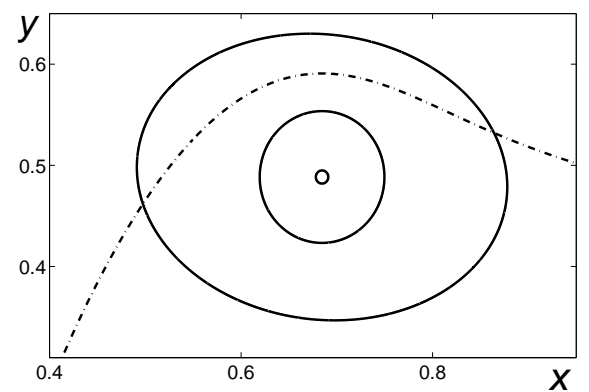

b)

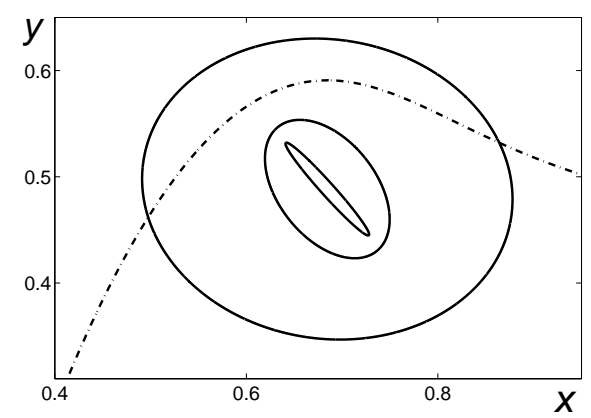

Figure 5. Separatrix (dash-dotted line) and confidence ellipses for $\beta=0.3, P=0.9$, and $\varepsilon=0.02$. In each figure the largest ellipse corresponds to the system without control; a) for Control I providing $w=1$ (medium sized ellipse), and $w=0.01$ (small sized ellipse); b) for Control II providing $w=1$ (medium sized ellipse), and $w=0.45$ (small sized ellipse).

uncontrolled system. The rest elements have to satisfy the condition of positive definiteness of the matrix $W$ :

$$
w_{11}>0, \quad w_{11} w_{22}>w_{12}^{2}=\frac{s_{2}^{2}}{4 f_{21}^{2}} .
$$

Using (3.9), one can write an explicit representation for the elements of the feedback matrix $K=$ $\left[\begin{array}{ll}k_{11} & k_{12}\end{array}\right]:$

$$
\begin{gathered}
k_{11}=-f_{11}+\frac{f_{21} w_{11} w_{12}-0.5 s_{1} w_{22}}{w_{11} w_{22}-w_{12}^{2}}, \\
k_{12}=-f_{12}+\frac{0.5 s_{1} w_{12}-f_{21} w_{11}^{2}}{w_{11} w_{22}-w_{12}^{2}} .
\end{gathered}
$$

Let $w_{11}=w_{22}=w$. Note that the value $w$ can not be chosen as small as one desires because $w>\left|w_{12}\right|$. For $\beta=0.3$, the value $w_{12}=-0.44$. Here we can assign the non-diagonal matrix $W: W=\left[\begin{array}{cc}w & -0.44 \\ -0.44 & w\end{array}\right]$.

For $w=1$, the stochastic sensitivity matrix of the controlled system is

$$
W_{1}=\left[\begin{array}{cc}
1 & -0.44 \\
-0.44 & 1
\end{array}\right]
$$

and for $w=0.45$, the stochastic sensitivity matrix of the controlled system is

$$
W_{2}=\left[\begin{array}{cc}
0.45 & -0.44 \\
-0.44 & 0.45
\end{array}\right]
$$

In Fig. 5b, confidence ellipses corresponding to matrices $W_{1}$ (middle) and $W_{2}$ (small) are plotted. As one can see, the eccentricity of these ellipses is quite different but they entirely belong to the basin of attraction of the nontrivial equilibrium $M_{4}$.

Results of the direct numerical simulation of solutions of the stochastic closed-loop system (3.3),(3.4) for three different values $\beta$ are presented in Fig. 6. Here, time series of this closed-loop system are plotted by black color. As one can see, the regulator (3.4) provides small stochastic sensitivity $w=0.1$ and stabilizes the population system near non-trivial deterministic equilibrium $M_{4}$. It is worth noting that 
a)

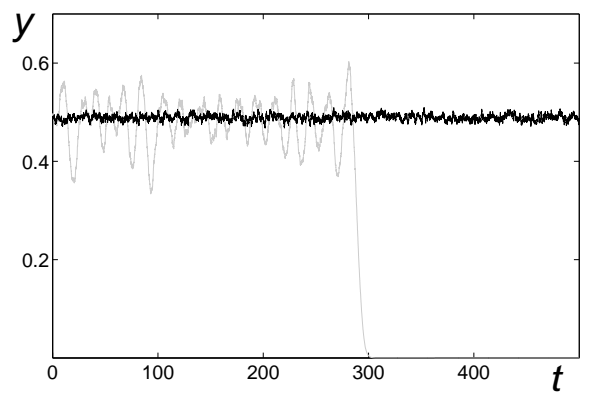

b)

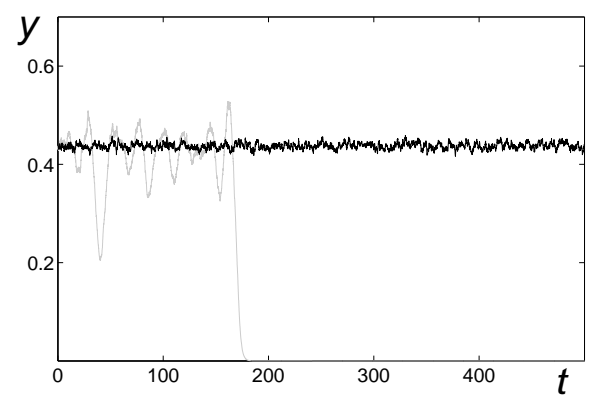

c)

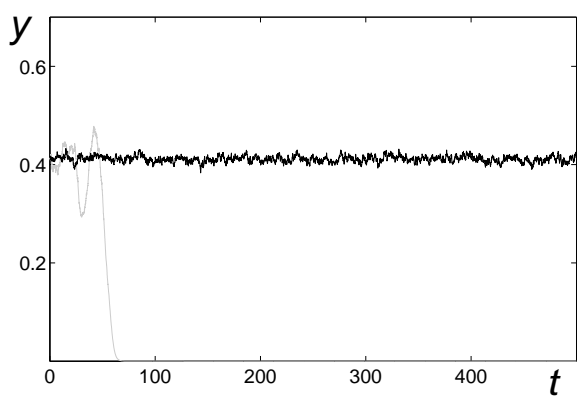

Figure 6 . Time series of the stochastic system $(\varepsilon=0.02)$ without control (grey) and with Control I (black) providing $w=0.1$ : a) for $\beta=0.3$; b) for $\beta=0.34$; c) for $\beta=0.36$.

this regulator allows us to stabilize even the unstable equilibrium (see Fig. $6 \mathrm{~b}$ for $b=0.34$ and Fig. 6c for $b=0.36)$.

Moreover, this regulator solves an important problem of the structural stabilization for the whole parametrical interval $0.3<\beta<0.38$ (Fig. 2c) and provides a small uniform dispersion of random states around $M_{4}$.

Thus, controlling these confidence ellipses we provide a stable coexistence of both species and prevent noise-induced extinction in this population model. Note that our approach based on SSF technique and method of confidence domains is quite universal and can be extended for the analysis and prevention of catastrophic shifts in more general models of complex ecological systems.

\section{Conclusion}

We study noise-induced phenomenon of the transitions from the coexistence to extinction for the randomly perturbed population model with the Allee effect. Our probabilistic analysis of this phenomenon is based on the stochastic sensitivity functions technique and confidence domains method. The threshold noise intensity that corresponds to the onset of such transitions is estimated by mutual arrangement of the confidence ellipses and separatrix. To prevent unwanted ecological shifts, we reduce a level of the stochastic sensitivity of the non-trivial equilibrium by the appropriate control. By controlling confidence ellipses, we provide a stable coexistence of both species and avoid noise-induced extinction. It is worth noting that our control method is robust and stable to the small errors in system parameters. 
Acknowledgements. This work was supported by the Ministry of Education and Science of the Russian Federation under the project N 315 .

\section{References}

[1] L. J. S. Allen. An introduction to the stochastic process with applications to biology. Pearson, Upper Saddle River, NJ, 2003.

[2] I. Bashkirtseva, L. Ryashko. Stochastic sensitivity of 3D-cycles. Math. Comp. Sim., 66 (2004), 55-67.

[3] I. Bashkirtseva, G. Chen, L. Ryashko. Stabilizing stochastically-forced oscillation generators with hard excitement: a confidence-domain control approach. Eur. Phys. J. B, 86 (2013), 437.

[4] A. A. Berryman. Stabilization or regulation: what it all means! Oecologia, 86 (1991), 140-143.

[5] A. A. Berryman. Limiting factors and population regulation. Oikos, 105 (2004), 667-670.

[6] A. J. Black, A. J. McKane. Stochastic formulation of ecological models and their applications. Trends in Ecology and Evolution, 27 (2012), 337-345.

[7] R. P. Blackshaw, S. V. Petrovskii. Limitation and regulation of ecological populations: a meta-analysis of Tipula paludosa field data. Math. Model. Nat. Phenom., 2 (2007), 46-62.

[8] S. R. Carpenter, L. H. Gunderson. Coping with collapse: ecological and social dynamics in ecosystem management. BioScience, 51 (2001), 451.

[9] O. Chichigina, D. Valenti, B. Spagnolo. A simple noise model with memory for biological systems. Fluctuation and Noise Letters, 5 (2) (2005), L243-L250.

[10] M. Dembo, O. Zeitouni. Large deviations techniques and applications. Jones and Bartlett Publishers, Boston, 1995.

[11] C. Folke, T. Hahn, P. Olsson, J. Norberg. Adaptive governance of social-ecological systems. Annual Review of Environment and Resources, 30 (2005), 441-473.

[12] M. I. Freidlin, A. D. Wentzell. Random perturbations of dynamical systems. Springer, New York, 1984.

[13] E. González-Olivares, A. Rojas-Palma. Allee effect in Gause type predator-prey models: existence of multiple attractors, limit cycles and separatrix curves. A Brief Review. Math. Model. Nat. Phenom., 8 (2013), 143-164.

[14] C. S. Holling. The functional response of predator to prey density and its role in mimicry and population regulation. Mem. Entomol. Soc. Canada, 45 (1965), 1-60.

[15] G. N. Mil'shtein, L. B. Ryashko. A first approximation of the quasipotential in problems of the stability of systems with random non-degenerate perturbations. J. Appl. Maths. Mechs. 59 (1995), 47-56.

[16] S. Petrovskii, A. Morozov, B. -L. Li. Regimes of biological invasion in a predator-prey system with the Allee effect. Bull. Math. Biol., 67 (2005), 637-661.

[17] L. Ridolfi, P. D’Odorico, F. Laio. Noise-induced phenomena in the environmental sciences. Cambridge University Press, Cambridge, 2011.

[18] M. Rietkerk, S. C. Dekker, P. C. de Ruiter, J. van de Koppel. Self-organized patchiness and catastrophic shifts in ecosystems. Science, 305 (2004), 1926-1929.

[19] L. B. Ryashko, I. A. Bashkirtseva. On control of stochastic sensitivity. Automation and Remote Control, 69 (2008), 1171-1180.

[20] L. Ryashko, I. Bashkirtseva. Stochastic sensitivity analysis of noise-induced excitement in a prey-predator plankton system. Frontiers in Life Science, 5 (2011) 141-148.

[21] M. Scheffer, S. Carpenter, J. A. Foley, C. Folke, B. Walker. Catastrophic shifts in ecosystems. Nature, 413 (2001), 591-596.

[22] B. Spagnolo, D. Valenti, A. Fiasconaro. Noise in ecosystems: a short review. Math. Biosci Eng. 1 (2004), $185-211$.

[23] D. Valenti, L. Schimansky-Geier, X. Sailer, B. Spagnolo. Moment equations for a spatially extended system of two competitive species. Eur. Phys. J. B 50 (2006), 199-203.

[24] D. Valenti, G. Augello, B. Spagnolo. Dynamics of a FitzHugh-Nagumo system subjected to autocorrelated noise. Eur. Phys. J. B, 65 (2008), 443-451. 